\title{
A REVIEW OF RECENT ACTIVITIES IN THE NASA CELSS PROGRAM
}

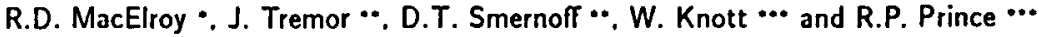 \\ - Planetary Biology Branch. Ames Research Center. Moffett Field. CA 94035 \\ * Complex Systems Research Center. University of New Hampshire. Dover. NH 03824 \\ *.* Biomedical Operations and Research Office, Kennedy Space Center, FL 32899
}

\begin{abstract}
A CELSS (Controlled Ecological Life Support System) is a device that utilizes photosynthetic organisms and light energy to regenerate waste materials into oxygen and food for a crew in space. The results of theoretical and practical studies conducted by investigators within the CELSS program suggest that a bioregenerative life support system can be a useful and effective method of regenerating consumable materials for crew sustenance. Experimental data suggests that the operation of a CELSS in space will be practical if plants can be made to behave predictably in the space environment. Much of the work currently conducted within the CELSS program centers on the biological components of the CELSS system. The work is particularly directed at ways of achieving high efficiency and long term stability of all components of the system. Included are explorations of the conversion of non-edible cellulose to edible materials, nitrogen fixation by biological and chemical methods. and methods of waste processing. It is the intent of the presentation to provide a description of the extent to which a bioregenerative life support system can meet the constraints of the space environment. and to assess the degree to which system efficiency and stability can be increased during the next decade.
\end{abstract}

\section{INTRODUCTION}

The Controlled Ecological Life Support System (CELSS) program is a research effort of the National Aeronautics and Space Administration (NASA) that has the goal of providing the research and technology base required to develop a bioregenerative life support system for use in extraterrestrial environments. Such environments include orbiting space stations. the lunar surface, transits to the outer planets, and bases on Mars and the asteroids. Recently, the National Commission on Space released its report /1/ which contains an exhilarating plan for American space exploration during the next half-century. The Commission recognized seven critical technologies that are essential for achieving their proposed space efforts; one of the seven is the development of long duration "closed-ecosystems". The approach of the CELSS program to fulfill this need is to explore first the development of a practical ground-based bioregenerative life support system. usable in the relatively near term. but to maintain close and active involvement with the planners who are developing concepts for larger. more complex systems /2/ suitable for colonies on the Moon or other planets /3.4/.

Bioregeneration is a feasible method of life support for those missions with large crews, or that are long-term and that cannot be resupplied easily or economically /5/. The CELSS concept is based on the natural processes of recycling that occur in the terrestrial environment. It is assumed that by artificially creating similar cycles of materials within small, closed systems. a usable ecosystem can be produced which is capable of supporting a crew. However, a CELSS is not directly analogous to the terrestrial ecosystem because its volume, and the sizes of its materials reservoirs are minute, and the rates of material cycling are much faster $/ 6 /$. Such characteristics dictate that the system be highly engineered and controlled.

A very large amount of scientific investigation and technological development will be required before a CELSS can be built for use in space. However, the directions that investigations must follow are reasonably clear, and such work is proceeding at laboratories around the world. The CELSS program has recently begun to explore the problems associated with scaling-up laboratory studies to sizes appropriate for the support of one person /7.8/. These larger scale investigations will utilize data generated in continuing laboratory studies, but they will also allow assessment of practical problems of life support. This paper will outline current views of critical scientific issues and will summarize plans for investigating the problems associated with large human-sized CELSS facilities. This COSPAR Workshop also includes expositions of recent data and concepts by investigators of the NASA CELSS program, on the growth of higher plants for CELSS (presented by F. Salisbury and by T. 
Tibbitts): on the use of algae in a CELSS (presented by Packer, by Radmer. and by Smernoff), and on food processing problems (presented by Karel).

\section{RESEARCH ACTIVITIES AND DRIVERS}

Many of the basic CELSS science investigations deal with the transformation of materials. The areas of fundamental research are guided by the need to understand the effect of environmental parameters on materials transformations. Specific examples are the effect of atmospheric $\mathrm{CO}_{2}$ concentration on the growth rate of wheat plants (the transformation of $\mathrm{CO}_{2}$ to wheat kernels). or the effect of temperature on the conversion of waste materials to $\mathrm{CO}_{2}$. nitrogen compounds, and water (transformation of organic materials to $\mathrm{CO}_{2}$ and water).

CELSS basic science can be considered as falling into distinct research areas which require significantly different scientific approaches. These are:

- Ground based research

- Flight investigations

It is assumed that ground experimentation will precede all flight experiments, and that flight experiments will seek specifically to address only questions concerning the effects of the space environment: principally gravity forces different from terrestrial and an altered radiation environment. Both ground-based and flight research must accomodate investigations that use two different approaches:

- Single variable analysis

- System analysis

Single variable analysis involves the experiments in which all variables except one are held constant and response to the remaining variable is examined. An example is the exploration of plant growth as a function of light intensity, or temperature, or $\mathrm{CO}_{2}$ concentration.

System analysis explores the coupling effects of variables, and will be significant not only for understanding the operation of the complete mechanical operation of a CELSS, but also for understanding biological activities. For example, the rate of $\mathrm{CO}_{2}$ uptake by plants is coupled to temperature. light intensity. $\mathrm{CO}_{2}$ concentration. growth phase. humidity. day length. nutrient composition, and other variables. System analysis is important to all CELSS science because of the need to maintain stable operation of all systems and to maximize efficiency.

It is frequently observed that system functions, particularly complex ones in which all behaviors are not welldefined (e.g organisms) exhibit behaviors that are not predictable a priori / $9 /$. While such behaviors may present no practical difficulties to the operation of a CELSS, it is prudent to demand that such systems be observed carefully for indications of instability $/ 10 /$ and that methods for overcoming possible instabilities be evaluated.

\section{PRACTICAL ISSUES}

The fact that the CELSS program can identify an end-item - a CELSS operating in an extraterrestrial environment - provides a focus for the program's research. but also allows the introduction of constraints which effectively narrow the scope of the scientific investigations. The primary constraints reflect the fact that. no matter where the operating site of a CELSS is found (in space, or on the lunar or martian surfaces). the system must be constructed to function within the mass, volume and power limitations of space flight.

These fundamental constraints require the use of efficiency of operation as a primary measure of the practicality of components. As a consequence, certain of the applied science directions to be followed can be identified. For example, even crude evaluations of CELSS efficiency allow placement of subsystems for animal food production and for chemical synthesis of food at lower priority levels. compared to subsystems for higher plant or algae growth.

Another significant influence on the direction of scientific studies is the sequence that will be followed in the development of a CELSS. from laboratory investigations through ground-based system demonstrations. flight experiments. man-tested facilities. to the construction and flight of a CELSS module for use in space. At the present time this sequence assumes that after investigations of scale-up. and preliminary component integration. a manned test facility should be constructed. This would be followed by a human-scale. functional mock-up. followed in turn by a module for use in space. Concurrently with ground-based developments. experiments conducted in space will be devised to answer questions about the behavior of components and small systems in the space environment. 
Development of a fully operable CELSS useful in space is the ultimate goal of the CELSS program, however. it is very likely that technology that is fully developed at the component level may be found useful for flight before a CELSS is completed. Examples are devices for the growth of salad plants to supplement the diet of a space station crew, or the use of a super-critical water reactor for waste disposal or air regeneration in space station.

\section{CRITICAL SCIENTIFIC AND TECHNOLOGICAL RESEARCH ISSUES}

Several areas of investigation fall into the category of critical issues. primarily because solutions to these problems are essential for consideration of a CELSS as a usable system, and because current information is insufficient to evaluate them properly. Examples of such critical issues that require investigation are:

Efficiency: minimizing the mass, power and volume required for the growth of organisms to provide complete life support capabilities within the constraints of mission:

Stability: understanding the behavior of essential organisms to allow predictable reliability of system operation over time periods of 2 to 10 years:

Space radiation: evaluating and minimizing its effects on growing organisms:

Gravity: understanding its effects on growing organisms sufficiently to allow the operation of a CELSS, with the possibility of reasonable centrifuge assist, within the gravity environment of the mission.

Human Labor Requirements: reduction of human labor requirements to a minimum through the use of automation and robotics, and simplification of man-machine interactions through the use of expert systems and mathematical models predictive of future system states.

Each of these issues is discussed in more detail below with the intention of identifying areas which require technology development.

\section{Efficiency}

The projected efficiency of a CELSS in operation in space will depend on many factors. For example: the mass of the equipment required to operate the system: the mass of water required to operate the biological subsystems: the volume that the system occupies, and the extent to which volume can be reduced by packing: the extent to which materials can be recycled. thus reducing resupply or stowage requirements: the power demands of the system. and the extent to which electrical power requirements can be reduced by introducing alternate lighting systems.

One of the elements in a CELSS to which the system is most sensitive is the food production subsystem. As an example. current estimates /11/ suggest that 18 to $20 \mathrm{~m}^{2}$ of growing wheat, when harvested and planted daily. will produce enough edible biomass to supply 2800 calories per day for human consumption. If the area required could be reduced to $15 \mathrm{~m}^{2}$, a reduction of 17 to $25 \%$. the mass of plant growth equipment, water, lighting equipment including solar panel equipment. and heat rejection equipment. could also be reduced proportionately. Alternatively, the amount of food available for crew use could be increased by the same amount. It is thus of great interest to explore various means to increase food production efficiency.

Efficiency increases in food production may be sought in many areas, including increasing the ratio of edible to non-edible biomass of plants: increasing the yield of individual plants: selecting highly productive crop plants: decreasing the time required for plant maturation; and converting inedible biomass to edible materials. Increases in photosynthetic efficiency may be useful, although the gains in system efficiency would be less marked than for other improvements. It should be noted that comments made by scientists from the Soviet Union suggest that the productivity of wheat can be increased. through selection of cultivars and identification of optimal environmental conditions. to a level equivalent to use of only 6 to $7 \mathrm{~m}^{2} / 12 /$.

While efficiency is currently measured in laboratory settings, using small plots, or small numbers of plants, there is a likelihood that much larger planting areas may be required in an operating CELSS. It is of considerable interest. therefore, that an increased scale of operation be explored. and that larger. integrated systems be tested.

\section{$\underline{\text { Stability }}$}

The concept of stability has both simple and complex aspects. Applied to a working system. the concept implies that the system will function. with proper maintenance, for as long as is necessary. Mechanical systems generally have the advantage that they can be turned on or off as appropriate: however, some mechanical systems must 
operate continuously and indefinitely. An example of the latter might be the cooling system of a nuclear power plant. To address the reality that a failure might occur requires introduction either of redundancy or some reliable measure of failure rate that can be factored into the estimate of reliability that is required.

Biological systems are also referred to as "stable". but the meaning is slightly different because it includes the knowledge that a biological system (perhaps a single organism) will age and will enter into a sequence of "stages" in its life cycle. Groups of organisms (fields of wheat. forests) are recognized as stable within well defined limits (e.g. until maturity). From the perspective of ecology. stability is a term that may be applied to a group of very diverse organisms, ranging from microbes to mammals. that are defined as members of an ecosystem. but again with the recognition that the term also includes the concept of succession of species. variations in populations within limits, and possibly as describing stages within evolutionary sequences.

Stability in a CELSS refers to the function of the system. The system is a complex one, and includes mechanical. chemical, physical, and biological components. By far, the components of such a system that are potentially the most unpredictable, unreliable and unstable are the biological ones. Recognition of this issue calls for thorough consideration of CELSS stability as a major research issue.

Of particular significance is the reliable and predictable growth of crops. A plant operates as a system that. over time, develops through what is usually seen as a sequence of phases, but what is, in fact, a continuum of many activities. Many of the activities can be observed to develop function. sustain the function for a period of time, and then cease the function. In higher plants the key to the sequence of these functions seems to lie in the major process of reproduction. Each of the processes are directly or indirectly affected by the environmental conditions.

The purpose of the CELSS research program is not to understand in detail the individual functions of a plant: rather, it is to assure that the functions occur in a reliable and predictable way. The hypothesis that develops from such considerations is that by maintaining all environmental conditions at known and appropriate levels at all times, the use of plants as the prime elements of a CELSS can allow stable, predictable and reliable system operation.

The basis for this hypothesis is that each of the functions of a plant can be considered to operate as a nonbiological chemical reaction: when reactants are supplied at a constant rate, and products are removed at a constant rate, the reaction will proceed in a predictable fashion.

Mating biological systems. containing their own innate control mechanisms (and that can be manipulated with generally unknown consequences). with physical and chemical systems (that can be manipulated with known consequences) will be difficult, but not impossible. It is expected that understanding the extent of the problem. which is one of the goals of CELSS science. will permit practical solutions to potential problems.

\section{Space radiation and Gravity}

The effect of continuous. accumulating. and increased levels of radiation on seed intended for establishing crops is presently unknown. It can be anticipated that extensive investigations must be done to establish that seed grown in one crop is suitable for planting a second. The doses of radiation will vary significantly with the mission: a low Earth orbit space station CELSS will be subject to less damaging radiation than will one in geosynchronous orbit. or a CELSS on the lunar surface. Protection of growing plants from radiation is possible. but the increased mass necessary for radiation absorbers must be weighed against the detrimental effects. Initially it is likely that planting seed for a CELSS will be grown on Earth and transported to space in radiation absorbing containers.

The combined effects of radiation and micro-gravity has been suggested for study in a group of organisms. and plants may offer a good study vehicle. The effect of lowered gravity on plant and crop growth is perhaps the most important CELSS-in-space issue that should be studied in the next decade. The issues involved are complex and will be studied in concert with wider investigations of gravitational biology and with NASA's Space Biology program. The interactive influence of radiation must also be explored.

\section{Human Labor Requirements}

The primary human labor requirement in a CELSS will be associated with plant cultivation. Most of the activities. however. can be automated. including planting and harvesting of crops. Food processing and preparation are also activities that can be extensively automated. In some cases, automation will be relatively easily achieved: in other cases, considerable data analysis will be needed, as will the introduction of robotic mechanisms. These issues are under study within the CELSS program /13/. 
Scientific investigations will continue to focus on methods of increasing the efficiency of higher plant growth. For example, new varieties of crops that mature much more rapidly will be tested: lighting and temperature regimes that promote growth rates will be explored: and environmental regimes that enhance growth will be studied under well-controlled conditions. Waste management and food processing investigations will become of greater importance as the CELSS research program continues.

The NASA CELSS program has recently begun to develop what it terms a Breadboard Project. The project is NASA's initial approach to assessing problems associated with large bioregenerative life support systems. The CELSS Breadboard Project will provide hardware, systems, and techniques for the production of biomass and oxygen, the preparation of food, and the processing of waste in a controlled recycling system. This project will fabricate. test, and operate a breadboard facility to accomplish a proof-of-concept evaluation. It will characterize system operations and mass and energy budgets.

The project will center around a modified steel chamber. $3.6 \mathrm{~m}$ in diameter by $6.7 \mathrm{~m}$ tall (volume: approximately $68 \mathrm{~m}^{3}$ ) for the growth of plants. The chamber will be placed in operation in late 1986 and will initially study the growth of wheat with the intention of determining how efficiencies obtained in the laboratory can be duplicated on a larger scale. The chamber will provide a unique facility for integrating and testing technologies. such as hydroponic plant culture systems. food harvesting and processing equipment. atmospheric and water regeneration techniques, and lighting systems.

The effort is proceeding under the guidance of a number of CELSS scientists, expert in the fields of food production, food processing, and waste management. As the project develops. it will approach regeneration by closure of the chamber to the entrance and exit of materials, and by increasingly efficient recycling materials. The experience gained in the development and use of the facility will factor into the design of a manned verification unit and eventually into flight equipment.

This combined approach, including research and breadboard tests, will assure that technical questions raised over the course of the program will have a proper scientific base in their answers.

\section{REFERENCES}

1. Pioneering the Space Frontier. Report to the President by the National Commission on Space, Bantam Books. New York. pp. 10-11, 1986.

2. The Biosphere Catalog. Snyder. T.P., ed., Synergetic Press. London, 254p. 1985.

3. MacElroy. R.D.. Klein. H.P. and Averner. M.M.. "The evolution of CELSS for Lunar bases", in Lunar Bases and Space Activities of the 21st Century. W.W. Mendell, ed.. Lunar and Planetary Institute. Houston. pp. 623-634. 1986.

4. MacElroy. R.D. and Smernoff. D.T.. Use of Martian Resources in a Controlled Ecological Life Support System. J. Brit. InterPlanetary Soc., in press, 1986.

5. Gustan. E. and Vinopal, T.. CELSS Transportation Analysis. NASA CR 166420. 1982.

6. MacElroy. R.D., and Bredt, J., "Current concepts and future directions of CELSS" in Adv. in Space Research. H. Oser. et al. eds., Vol. 4. Number 12. Pergamon Press. pp. 221-230, 1984.

7. Knott. W.M. "Plans for CELSS test bed project", in Controlled Ecological Life Support Systems: CELSS'85 Workshop. R.D. MacElroy. et. al. eds., NASA TM-88215. pp. 109-118, 1986.

8. Prince. R.P. and Knott. W.M.. "Plant growth chamber ' $M$ ' design" in Controlled Ecological Life Support Systems: CELSS'85 Workshop. R.D. MacElroy, et. al. eds.. NASA TM-88215. pp. 119-128. 1986.

9.Stahr. J.D.. Auslander. Spear. R.C. and Young. G.E.. An approach to the preliminary evaluation of CELSS scenarios and control strategies. NASA CR-166368, 1982.

10. Young. G. A design methodology for non-linear systems containing parameter uncertainty: application of non-linear controller design. NASA CR-166358, 1982.

11. Bugbee. B.G. and Salisbury. F.B.. "Studies on Maximum Yield of Wheat for the Controlled Environments of Space". in Controlled Ecological Life Support Systems: CELSS'85 Workshop. R.D. MacElroy, et. al. eds.. NASA TM-88215. pp. 447-486. 1986.

12. Polonskiy. V.I. and Lisovskiy. G.M. Techniques for creating rapid growth strains of wheat for human life support systems. Kosmicheskaya Biologiya i Aviakosmocheskaya Meditsina, 20(2):96, 1986. [Translation of an abstract describing the paper cited]

13. Oleson. M.W. and R.L. Olson. Controlled Ecological Life Support Systems (CELSS) Conceptual Design Option Study. NASA CR-177421, 1986. 